\title{
The Optimal Design of Hydraulic Fracture Parameters in Fractured Gas Reservoirs with Low Porosity
}

\author{
Xiuling Han $^{1}$, Fujian Zhou $^{2}$,Chunming Xiong ${ }^{1}$,Xiongfei Liu $^{2}$, Xianyou Yang ${ }^{2}$ \\ Xiuling Han \\ Research Institute of Petroleum Exploration \& \\ Development \\ RIPED, PetroChina \\ Beijing, China \\ hanxiuling496@yahoo.cn \\ Fujian Zhou \\ Research Institute of Petroleum Exploration \& \\ Development \\ RIPED-Langfang, PetroChina \\ Beijing, China \\ zhoufj@petrochina.com.cn
}

\begin{abstract}
A new composite reservoir simulation model was developed to optimize the hydraulic fracture length and conductivity for guiding fracturing designs. The model is made of inner part and outer part. The inner part is dual-porosity and dual-permeability system, and the other is single porosity system. Based on the model, the effect of reservoir parameters, hydraulic fractures parameters, and non-Darcy flow effects on gas production were studied. The research shows that the natural fracture permeability and density are the most influential parameters; a relative long fracture with high hydraulic fracture conductivity is required for a high production rate due to non-Darcy flow effects. A shorter primary fracture is better in a gas reservoir of high natural density. The composite model represents the flow characteristic more accurately and provides the optimal design of fracturing treatments to obtain an economic gas production.
\end{abstract}

Keywords-Optimal design; Hydraulic fracture; composite model; non-Darcy; naturally fractured reservoir

\section{INTRODUCTION}

Hydraulic fracturing is the most effective technology for unconventional gas reservoirs to obtain a commercial gas flow rate at present. The hydraulic fracture length and conductivity are main parameters to optimize in a design of hydraulic fracturing technology. Numerical simulations provide a better way to determine hydraulic fracture parameters over other methods.

There are hydraulic fractures, natural fractures and the matrix to be considered during numerical simulations. Dual porosity (DP) models/ dual porosity and dual permeability (DPDK) models ${ }^{[1-7]}$, single porosity models ${ }^{[8]}$, and discrete models ${ }^{[9-11]}$ are the most commonly used methods for naturally fractured reservoirs. Single porosity models cannot represent the interaction between the fracture and the matrix, and discrete models are time-consuming in setting up hydraulic and natural fractures and of high computational cost. Also DP/DPDK needs large grid to simulate and long time to run.

In this paper, a simplified composite model is proposed to optimize hydraulic fracture parameters. The flowing area of the gas reservoir is divided into two parts, the inner parts and outer parts. The inner part of the model is a complex fracture network, including hydraulic fractures and induced natural fractures as in Fig. 1. DP/DPDK is used to describe the flowing characteristic between the matrix and the fracture. It treats the porous system as two distinct continua, the fracture of high flowing capacity but low storage capacity and the matrix of low flowing capacity but high storage capacity. Fractures are highly interconnected fed by numerous matrix blocks. The outer part of simulation model is a single porosity model where micro-fractures are less developed than the inner part. A high equivalent permeability is used to describe natural fractures, and it may be interpreted as a single porosity medium. The composite model is used to optimize hydraulic fracture length and conductivity by considering natural fracture (NF) density, natural fracture permeability, and non-Darcy effects on gas productivity.

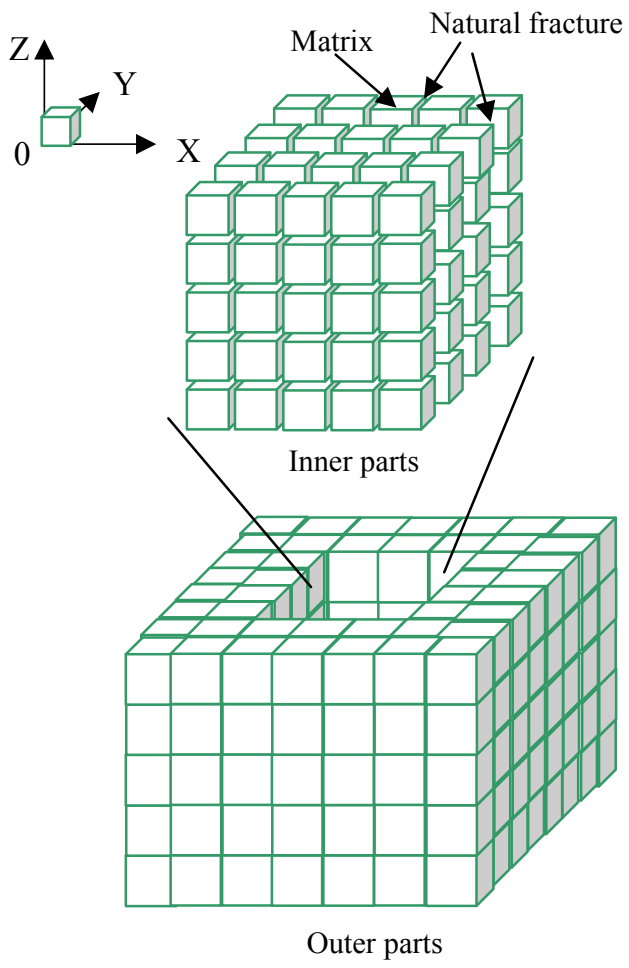

Figure 1. Schematic of the composite reservoir model 


\section{NUMERICAL MODEL DESCRIPTION}

\section{A. Assumptions}

Formulation of the governing equations is based on the following assumptions:

- Darcy's law describes the multiphase flow in the matrix, and non-Darcy flow in the fracture,

- Considering water phase and gas phase,

- The rock is slightly compressible and immobile,

- Water is slightly compressible,

- And the hydraulic fracture is throughout to penetrate the whole layer and has a finite conductivity, fracture failure is considered, and capillary in the fracture system is neglected.

\section{B. Governing Equations}

In the composite model, the grid of the inner parts is divided into two partitions, one for the fracture and another for the matrix. The grid in the outer parts is only the matrix with an equivalent permeability. Equations of the inner parts can be expressed as

Fracture system:

$$
\frac{\partial\left(\rho_{l f} \phi_{f} S_{l f}\right)}{\partial t}+\rho_{l f} \frac{K_{f} K_{l r f}}{\mu_{l}} \operatorname{div}\left(\delta \operatorname{grad} P_{l f}\right)-\tau_{l m-f}+q_{l f}=0
$$

Matrix system:

$\frac{\partial\left(\rho_{l m} \phi_{m} S_{l m}\right)}{\partial t}+\rho_{l m} \frac{K_{m} K_{l r m}}{\mu_{l}} \operatorname{div}\left(\operatorname{grad} P_{l m}\right)+\tau_{l m-f}+q_{l m}=0$

Transfer function:

Shape factor ${ }^{[2]}$ :

$$
\tau_{l m-f}=\frac{\sigma \delta K_{l m} K_{l r m}}{\mu_{l}}\left(P_{l m}-P_{l f}\right)
$$

$$
\sigma=4\left(\frac{1}{L^{2} x}+\frac{1}{L^{2} y}+\frac{1}{L^{2} z}\right)
$$

The governing equation of outer part is commonly used in conventional reservoirs as in (5).

$$
\frac{\partial\left(\rho_{l m} \phi_{m} S_{l m}\right)}{\partial t}+\rho_{l m} \frac{K_{m} K_{l r m}}{\mu_{l}} \operatorname{div}\left(\operatorname{grad} P_{l m}\right)+q_{l m}=0
$$

where

$l \quad=$ gas phase or water phase

$\phi_{\mathrm{m}}=$ matrix porosity, $\mathrm{f}$

$\phi_{f} \quad=$ fracture porosity, $\mathrm{f}$

$P_{\mathrm{m}}=$ matrix pressure, $\mathrm{MPa}$

$P_{f} \quad=$ fracture pressure, $\mathrm{MPa}$

$K_{\mathrm{m}}=$ matrix permeability, $10^{-3} \mu \mathrm{m}^{2}$

$K_{f}=$ fracture porosity, $10^{-3} \mu \mathrm{m}^{2}$

$q_{l m}=$ production from the matrix, $\mathrm{m}^{3} / \mathrm{d}$

$q_{\text {lf }}=$ production from the fracture, $\mathrm{m}^{3} / \mathrm{d}$

$\delta=$ non-Darcy flow factor, the value of 1 means Darcy flow, and less than 1 means non-Darcy flow, $\mathrm{f}$
$\mathrm{Lx}, \mathrm{Ly}$, and $\mathrm{Lz}=$ length of matrix blocks at the direction of $\mathrm{X}, \mathrm{Y}$, and $\mathrm{Z}, \mathrm{m}$

The outer boundary condition is closed; the inner boundary is specified constant pressure or production rate. The pressure and flowing production rate is equal not only at the interface of the inner part and the outer part but also at the matrix and the fracture. Finite difference method is used to solve seepage flow equation, and Gauss-Seidel is adopted to solve the pressure of matrix and fracture at any time.

\section{CALCULATION OF MODEL PARAMETERS}

\section{A. Hydraulic fracture failure}

With the increase of production time after fracturing treatment, the fracture conductivity gradually reduces due to the decrease of pore pressure and increase of overburden pressure. According to analysis of field production effects and results of long-time conductivity laboratory experiment, the change of fracture conductivity is related to producing time as in (6).

$$
F_{c d}=F_{c d 0} e^{-b t}
$$

where $F_{c d}$ is fracture conductivity after treatment, $\mu \mathrm{m}^{2} \cdot \mathrm{cm}$; $F_{c d 0}$ is fracture conductivity at initial time after fracturing, $\mu \mathrm{m}^{2} \cdot \mathrm{cm} ; \mathrm{b}$ is the conductivity attenuation coefficient obtained from experiment, $\mathrm{f} ; \mathrm{t}$ is the producing time.

\section{B.Non-Darcy Flow Factor}

Non-Darcy flow factor describes a quantitative indication of the deviation of the flow behavior from Darcy's law.

$$
\delta_{\mathrm{x}}=\frac{-1+\sqrt{1+4 C_{1} C_{2} \beta \rho_{l}\left(\frac{K K_{\text {lrf }}}{\mu_{l}}\right)^{2} \frac{\partial P}{\partial \mathrm{x}}}}{2 C_{1} C_{2} \beta \rho_{l}\left(\frac{K K_{l \mathrm{lf}}}{\mu_{l}}\right)^{2} \frac{\partial P}{\partial \mathrm{x}}}
$$

where $C_{1}$ and $C_{2}$ are constants; $\beta$ is the non-Darcy coefficient obtained from experiment or empirical formula, as a function of reservoir permeability, porosity, multiphase, etc. Then the same method are applied to get $\delta_{\mathrm{y}}$ and $\delta_{\mathrm{z}}$.

\section{Productivity calculation}

The fluid from the reservoir into the well-bore consists of three parts: (1) flowing from matrix directly into the wellbore; (2) flowing from the natural fractures into the well-bore; (3) flowing from the hydraulic fracture into the well-bore.

$$
\begin{gathered}
Q_{l}=Q_{N f}+Q_{m}+Q_{H f} \\
Q_{H f}=\sum_{m=1}^{n}\left[q_{m}(i, j, k)+q_{f}(i, k)\right]
\end{gathered}
$$

where $Q_{l}$ is total production, $10^{4} \mathrm{~m}^{3} ; Q_{H f}$ is production flowing from the hydraulic fracture, $10^{4} \mathrm{~m}^{3} ; Q_{N f}$ is production flowing from the natural fractures, $10^{4} \mathrm{~m}^{3} ; Q_{m}$ is production flowing from the matrix; $m$ is the number of simulation grid, $\mathrm{m}=1,2, \ldots, \mathrm{n} ; \mathrm{n}$ is the simulation grid number of hydraulic fractures, f; $q_{m}(i, j, k)$ is the production 
flowing from the matrix, $10^{4} \mathrm{~m}^{3} ; q_{f}(i, k)$ is the production flowing from the natural fractures, $10^{4} \mathrm{~m}^{3}$.

\section{SIMULATION CALCULATION AND ANALYSIS}

To evaluate sensitivities of natural fracture density and permeability, non-Darcy flow effect and optimize the fracture length and conductivity, a base model was constructed in a low porosity and fractured gas reservoir. Grid cells in the outer parts are $50 \mathrm{~m}$ in length, $50 \mathrm{~m}$ in width, $10 \mathrm{~m}$ in height. The hydraulic fractures are explicitly represented by a grid with a width of $0.1 \mathrm{~m}$ in the inner grid cells as shown in Fig. 2. Other basic reservoir parameters are listed in Table 1.

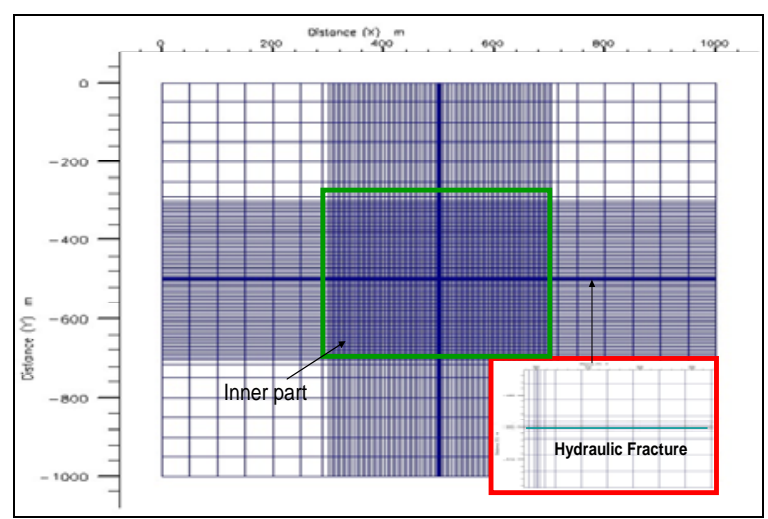

Figure 2. Grid division of the base model.

TABLE I. RESERVOIR PARAMETERS

\begin{tabular}{|c|c|}
\hline Reservoir area, $\mathrm{m}^{2}$ & $1000 \times 1000$ \\
\hline layer thickness, $\mathrm{m}$ & 40 \\
\hline Average matrix porosity, $\mathrm{f}$ & 0.08 \\
\hline Average fracture porosity, $\mathrm{f}$ & 0.006 \\
\hline Average NF permeability, $10^{-3} \mathrm{\mu m}^{2}$ & 1 \\
\hline Average matrix permeability, $10^{-3} \mathrm{\mu m}^{2}$ & 0.005 \\
\hline Reservoir pressure, $\mathrm{MPa}$ & 95 \\
\hline Conductivity attenuation coefficient, $\mathrm{f}$ & 0.0038 \\
\hline Bottom hole pressure, $\mathrm{MPa}$ & 85 \\
\hline Average NF density, $\mathrm{m}^{-1}$ & 1 \\
\hline
\end{tabular}

\section{A. Reservoir parameters effect}

The natural fracture makes great contributions to gas production before and after hydraulic fracturing. So a sensitivity study was performed on natural fracture density ranging from $0.5-2 \mathrm{~m}^{-1}$, natural fracture permeability raging from $0.25-10 \times 10^{-3} \mu^{2}$, NF porosity raging from $0.4 \%$ $0.8 \%$, the matrix porosity raging from $6 \%-10 \%$ and the matrix permeability $0.0005-0.05 \times 10^{-3} \mu \mathrm{m}^{2}$. Fig. 3 shows the impact of the reservoir parameters on the gas production performance.

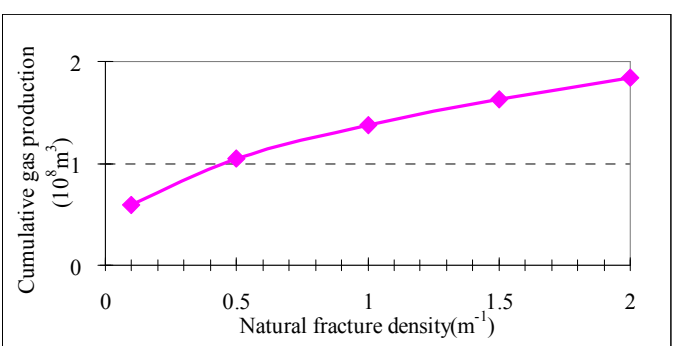

(a)Effect of NF density

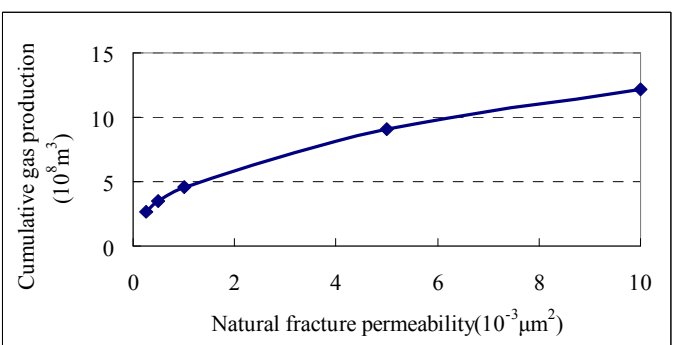

(b) Effect of NF permeability

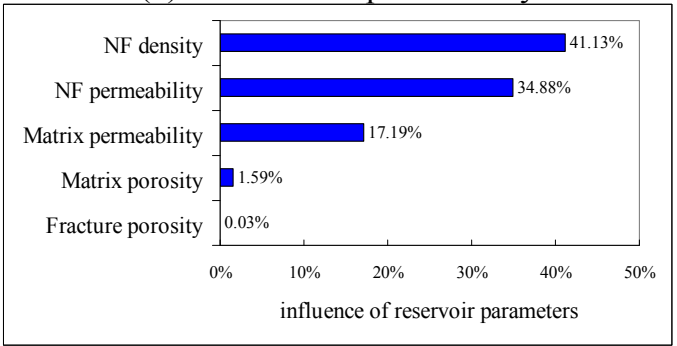

(c) Effect of main reservoir parameters

Figure 3. Effect of reservoir parameters on cumulative gas production.

Fig. 3 demonstrates that NF density and NF permeability are the most influential reservoir parameters. It can be confirmed from oilfield production. The gas production of field is higher when NF is well developed in reservoir ${ }^{[5]}$. In composite model, natural gas flows into wellbore from matrix and fracture simultaneously at the inner part. Therefore the effect of matrix permeability on gas production is relatively strong than the effect of matrix and fracture porosity.

\section{B. Hydraulic fracture parameters}

In fractured layers, since it is difficult to create a long primary hydraulic fracture with high fracture conductivity, fracture half length varies from 50 to $200 \mathrm{~m}$, and the hydraulic fracture conductivity is limited from 5 to $30 \mu \mathrm{m}^{2} \cdot \mathrm{cm}$. The impact of hydraulic fracture parameters on the cumulative gas production is shown in Fig. 4. The effect of hydraulic fracture half length on the drainage area can be seen in Fig. 5. With the increase of fracture half length, cumulative gas production increases, but the increase tends to slow down when fracture conductivity is less than 10 $\mu \mathrm{m}^{2} \cdot \mathrm{cm}$. The longer the hydraulic fracture half length, the 
higher fracture conductivity is needed to deliver more gas into the well-bore when the primary fracture conductivity is greater than $10 \mu \mathrm{m}^{2} \cdot \mathrm{cm}$. The effect of natural fracture density on the optimization of primary fracture length is shown in Fig. 6.

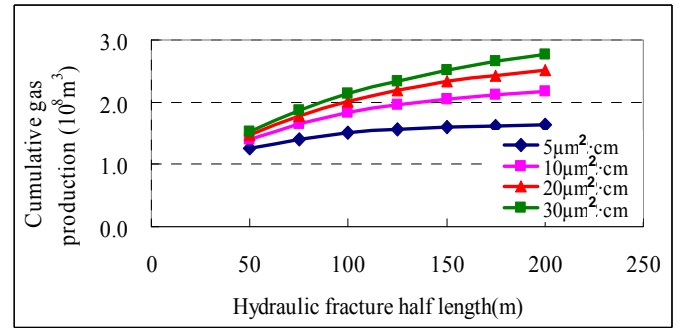

Figure 4. Effect of hydraulic fracture parameters on cumulative gas production.

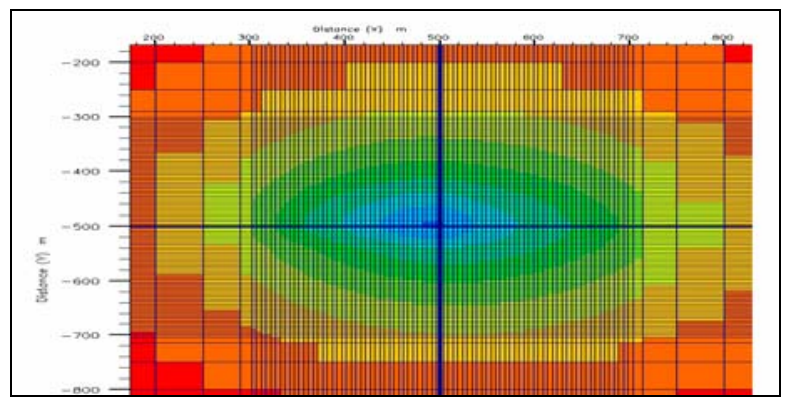

Figure 5. Effect of hydraulic fracture half length on the drainage area

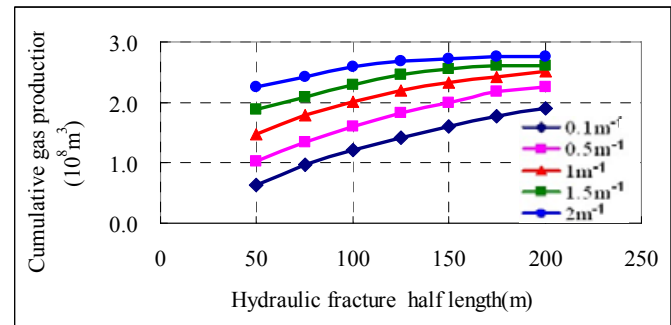

Figure 6. Effect of natural fracture desity with different hydraulic fracture length.

Fig. 6 shows that natural fracture density has great impact on the optimization of primary hydraulic fracture length. For a high NF density, the effect of primary fracture length decreases, so a shorter primary fracture length can satisfy an economic production. When NF is less developed in a gas reservoir, the longer the primary hydraulic fracture, the higher gas production are obtained.

\section{Non-Darcy flow effects}

Fig. 4 demonstrates that increasing primary fracture length requires increasing fracture conductivity to achieve more economical productivity possibly. Non-Darcy flow effects reduce the effective permeability of the proppant, so the effective fracture conductivity decreases in gas wells.
The impact of non-Darcy flow on cumulative gas production is shown in Fig. 7.The impact of non-Darcy flow is less with a low value of the fracture conductivity than high fracture conductivity, so a higher permeability of the proppant in the fracturing treatment design can compensate for the permeability reduction caused by non-Darcy flow.

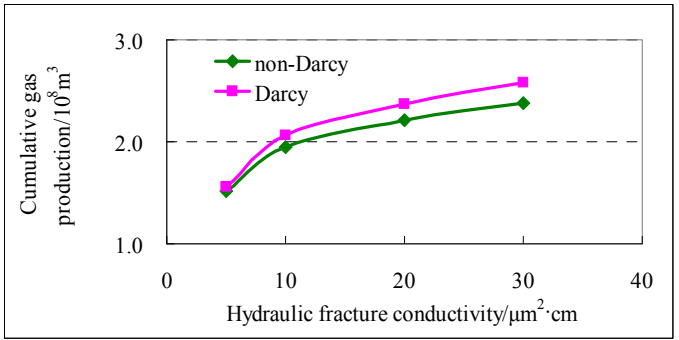

Figure 7. Effect of non-Darcy flow effects on cumulative gas production.

\section{SUMMARY}

A composite gas reservoir model was developed to optimize hydraulic length and conductivity considering nonDarcy flow and hydraulic fracture failure. The gas production after fracturing can be represented well and provides a preferable foundation for the design of hydraulic fracture treatments.

The NF permeability and NF density are the most influential ones of reservoir parameters. Hydraulic fracture length and hydraulic fracture conductivity are the important factors in design of hydraulic fracturing. A higher gas production can be obtained with the increase of fracture conductivity when the primary fracture is relative long. If NF is well developed with strong natural fracture strength, a shorter primary fracture can yield a high gas production. A longer hydraulic fracture is required for economic production rate in a reservoir with less NF density. The optimization of primary hydraulic fracture length and fracture conductivity has a closed relationship with NF density.

Non-Darcy flow effects reduce effective fracture conductivity in gas wells. To obtain a high gas production, the fracture conductivity should be increased accordingly considering non-Darcy flow effects at the design of hydraulic fracture treatments.

\section{REFERENCES}

[1] J.E.Warren , P.J.Root, "The behavior of naturally fractured reservoirs," paper SPE426,SPEJ, 1963.

[2] H.Kazemi "Pressure transient analysis of naturally fractured reservoir with uniform fracture distribution,"paper SPE 2156, SPEJ, 1969.

[3] T.M.Al-Shaalan, L.S.K. Fung, and A.H.Dogru, "A Scalable Massively Parallel Dual-Porosity Dual- Permeability Simulator for Fractured Reservoirs with Super-K Permeability," paper SPE84371, presented at the SPE Annual Technical Conference and Exhibition, 2003.

[4] M.G.Correia, J.Denis, and S.Marcos, "Sensitivity for Dual Porosity and Dual permeablity Systems to Small and Large Scale Heterogeneities in a Naturally Fracturally Fractured Carbonate Reservoir," paper SPE143149, presented at the Brasil Offshore Conference and Exhibition, 2011. 
[5] X. Zhang, C. Du, and M.Crick, A.Harikesavanallur "Sensitivity Studies of Horizontal Wells with Hyddraulic Fractures in Shale Gas Reservoirs," IPTC 13338,2009.

[6] L. James, D. Mike,and X. Zhang, "Critical Evaluation of Shale Gas Reservoir Simulation Approaches: Single-Porosity and Dual-Porosity Modeling," Paper SPE 141756, presented at the Middel East Unconvetional Gas Conference and Exhibition, 2011.

[7] B.Ramires, H. Kazemi, and E.Ozkan, "Non-Darcy Flow Effects in Dual-Porosity, Dual-Permeability Naturally Fractured Gas Condensate Reservoirs," paper SPE109295, SPE Annual Technical Conference and Exhibition, 2007.

[8] J.W. Wang, Y.Liu, "Simulation Based Well Performance Modeling in Haynesville Shale Reservoir," Paper SPE 142740, presented at the Production and Operations Symposium, 2011.
[9] K. Suksang, A. Datta-Gupta, W. Hohn lee, "Impact of Natural Fractures in Drainage Vloume Calculations and Optimal Well Placement in Tight Gas Reservoirs," Paper SPE 144338, presented at the 2008 SPE Shale Gas Production Conference, 2008.

[10] B. Gong, Q. Guan, S.Y. Yuan, "Detailed Modeling of the Compaex Fracture Network of Shale Gas Reservoirs," Paper SPE 142705, presented at the 2010 SPE Middle East Uncoventional Gas Conference, 2010.

[11] M. Karimi-Fard, L.J.Durlofskky, K.Aziz, "An efficient discrete fracture model applicable for general-purpose reservoir simulators," Paper SPE 88812, SPEJ, 2004. 